\title{
Fast Formation of Opal-like Columnar Colloidal Crystals
}

\author{
David van der Beek,* Paul B. Radstake, Andrei V. Petukhov, and Henk N. W. Lekkerkerker \\ Van't Hoff Laboratory for Physical and Colloid Chemistry, Utrecht University, Padualaan 8, \\ 3584 CH Utrecht, The Netherlands
}

Received May 3, 2007. In Final Form: July 29, 2007

\begin{abstract}
We demonstrate that highly polydisperse colloidal gibbsite platelets easily form an opal-like columnar crystal with striking iridescent Bragg reflections. The formation process can be accelerated by orders of magnitude under a centrifugation force of $900 \mathrm{~g}$ without arresting the system in a disordered glassy phase. Using transmission electron microscopy and small-angle X-ray scattering techniques, we find that the forced sedimentation is accompanied by particle size fractionation, leading to inversion of the iridescent colors. The relatively easy self-organization of the polydisperse colloidal particles into opal-like crystals may be explained on the basis of the observed particle fractionation and possibly also on hexatic-like ordering.
\end{abstract}

In the 1960s, Pense ${ }^{1}$ and Jones, Sanders, and Segnit ${ }^{2}$ recognized that opal consists of a regular packing of monodisperse amorphous silica spheres of $100-300 \mathrm{~nm}$ diameter. The relation between the particulate structure and the reflected colors of opal was described by Sanders. ${ }^{3}$ The fact that isodimensional colloidal particles can form crystal structures that exhibit Bragg reflection of visible light was established earlier for virus particles ${ }^{4,5}$ (Tipula iridescent virus, $130 \mathrm{~nm}$ diameter) and latex particles ${ }^{6}(150-500$ $\mathrm{nm}$ diameter). Following the discovery of the structure of opal, efforts to make synthetic opals in the laboratory ensued. ${ }^{7}$ Initially, attempts were made to synthesize colloidal silica spheres from

* To whom correspondence should be addressed. Present address: Albemarle Catalysts Company B.V., Nieuwendammerkade 1-3, 1022 AB Amsterdam, The Netherlands. E-mail: david.vanderbeek@albemarle.com.

(1) Pense, J. Elektronenmikroskopischer Beitrag zur Optik der Edelopale Jahrestag der Deutschen und Österreichischen Mineralogischen Gesellschaft; Wien, 1963. For a description, see Baier, E. Experientia 1966, 22, 129.

(2) Jones, J. B.; Sanders, J. V.; Segnit, E. R. Nature 1964, 204, 990.

(3) Sanders, J. V. Nature 1964, 204, 1151.

(4) Williams, R. C.; Smith, K. M. Nature 1957, 179, 119.

(5) Klug, A.; Franklin, R. E.; Humphreys-Owen, S. P. F. Biochim. Biophys. Acta 1959, 32, 203.

(6) Alfrey, T.; Bradford, E. B.; Vanderhof, J. F.; Oster, G. J. Opt. Soc. Am. 1954, 44, 603. Fischer, E. W. Kolloid Z. 1958, 160, 120. Luck, W.; Klier, M.; Wesslau, H. Ber. Bunsen-Ges. Phys. Chem. 1963, 67, 75. Luck, W.; Klier, M. Wesslau, H. Naturwissenschaften 1963, 50, 485.

(7) Gaskin, A. J.; Darragh, P. J. Opaline Materials and Materials of Preparation. U.S. Patent 3,497,367, 1970. inorganic materials. It proved to be very difficult to create sufficiently monodisperse particles. (See Iler. ${ }^{8}$ ) The Stöber synthesis ${ }^{9}$ provided an easy and reproducible synthesis route to monodisperse colloidal silica spheres, and in 1979, Gilson succeeded in making synthetic opals. ${ }^{10}$ To obtain good-quality crystals, slow sedimentation (more than 1 year) of dilute silica dispersions yields the best results. ${ }^{11}$ However, if the size of the colloidal particles is extremely monodisperse as for the Tipula iridescent virus, a relatively rapid increase in concentration (centrifugation) still results in the formation of higly ordered crystals. ${ }^{5}$ Over the last 10 years, the focus of synthetic opals has been in the area of photonic band gap materials where inverse opals (consisting of air spheres) seem to hold great promise and are attracting a lot of attention. ${ }^{12}$

In addition to spheres, there are anisometric colloidal particles (rods and plates) that form iridescent ordered phases. The first example of an iridescent smectic phase was observed as early

(8) Iler, R. K. Nature 1965, 207, 472.

(9) Stöber, W.; Fink, A.; Bohn, E. J. Colloid Interface Sci. 1968, 26, 62.

(10) Gilson, P. J. Gemmol. 1979, 16, 494.

(11) Filin, S. V.; Puzynin, A. I.; Samoilov, V. N. Aust. Gemmol. 2002, 21, 7.

(12) Imhof, A.; Pine, D. Nature 1997, 389, 984. Wijnhoven, J. E. J. G.; Vos, W. L. Science 1998, 281, 802. Blanco, A.; Chomski, E.; Grabtchak, S.; Ibisate, M.; John, S.; Leonard, S. W.; Lopez, C.; Meseguer, F.; Miguez, H.; Mondia, J. P.; Ozin, G. A.; Toader, O.; van Driel, H. M. Nature 2000, 405, 437. 

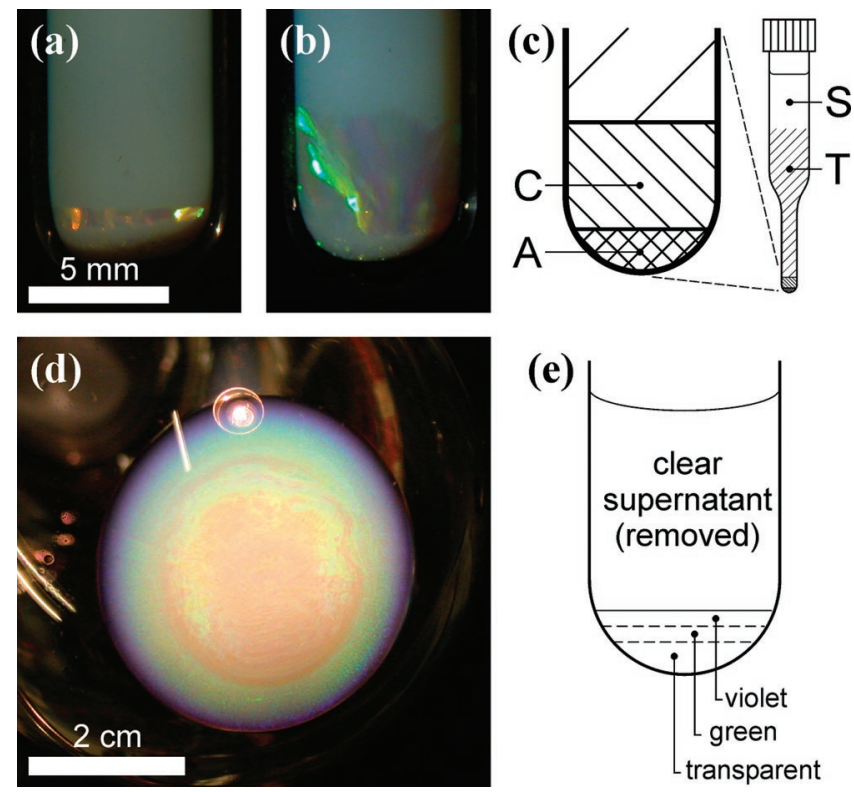

Figure 1. Iridescent columnar phase grown in a gravitational field. (a, b) Sample that has been standing for 2 and 4 years, respectively, at $1 g$. The scale bar pertains to both images. (c) Sketch of the crystallization tube and its layers: $\mathrm{S}$ is the clear supernatant, $\mathrm{T}$ is the turbid suspension, $\mathrm{C}$ is the well-ordered columnar crystalline layer, and A is the amorphous columnar crystal. At $900 \mathrm{~g}$, using a centrifuge, it takes only 1 day to create a columnar phase as shown in part d; its layers are sketched in part e.

as 1925 in suspensions of rodlike $\beta$-FeOOH. ${ }^{13}$ The resolution of the particulate structure came much later. ${ }^{14}$ Also, the rodlike particles of the tobacco mosaic virus ${ }^{15}$ form an iridescent smectic phase. ${ }^{16}$ Recently, smectic liquid-crystalline order with brightred Bragg reflections was discovered in suspensions of highly polydisperse goethite $(\alpha-\mathrm{FeOOH})$ rods. ${ }^{17} \mathrm{In}$ addition to smectic phases, there are lamellar phases of tungstic acid $\left(\mathrm{H}_{3} \mathrm{WO}_{4}\right)^{18}$ and phospate antimonite $\left(\mathrm{H}_{3} \mathrm{Sb}_{3} \mathrm{P}_{2} \mathrm{O}_{14}\right)^{19}$ in which iridescence has been observed.

Over the past few years, we have studied the liquid-crystalline phase behavior of colloidal gibbsite $\left.\left(\gamma-\mathrm{Al}(\mathrm{OH})_{3}\right)\right)$ platelets. ${ }^{20,21}$ We observed that suspensions of both sterically and chargestabilized gibbsite platelets at sufficiently high concentrations display beautiful iridescence. ${ }^{22,23}$ From high-resolution smallangle X-ray scattering, we have obtained unambiguous evidence that these iridescent phases have columnar structure with hexagonal intercolumnar ordering. ${ }^{24,25}$ This is quite remarkable because the platelets have a polydispersity of $19 \%(27 \%)$ in

(13) Zocher, H. Z. Anorg. Allg. Chem. 1925, 147, 91.

(14) Maeda, Y.; Hachisu, S. Colloids Surf. 1983, 6, 1. Maeda, Y.; Hachisu, S. Colloids Surf. 1983, 7, 357.

(15) Bernal, J. D.; Fankuchen, I. J. Gen. Physiol. 1941, 25, 111

(16) Oster, G. J. Gen. Physiol. 1950, 33, 445. Kreibig, U.; Wetter, C. A. Z. Naturforsch. 1980, 35C, 750 .

(17) Vroege, G. J.; Thies-Weesie, D. M. E.; Petukhov, A. V.; Lemaire, B. J.; Davidson, P. Adv. Mater. 2006, 18, 2565.

(18) Zocher, H.; Jacobsohn, K. Kolloid-Beih. 1929, 28, 167. Bergmann, P.; Low-Beer, P.; Zocher, H. Z. Phys. Chem. A 1938, 181, 301. Furasawa, K.; Hachisu, S. J. Colloid Interface Sci. 1968, 28, 167.

(19) Gabriel, J. C. P.; Camerel, F.; Lemaire, B. J.; Desvaux, H.; Davidson, P.; Batail, P. Nature 2001, 413, 504.

(20) van der Kooij, F. M.; Lekkerkerker, H. N. W. J. Phys. Chem. B 1998 102,7829 .

(21) van der Beek, D.; Lekkerkerker, H. N. W. Europhys. Lett. 2003, 61, 702

(22) van der Kooij, F. M.; Kassapidou, K.; Lekkerkerker, H. N. W. Nature 2000, 406, 868 .

(23) van der Beek, D.; Lekkerkerker, H. N. W. Langmuir 2004, 20, 8582.

(24) van der Beek, D.; Petukhov, A. V.; Oversteegen, S. M.; Vroege, G. J.; Lekkerkerker, H. N. W. Eur. Phys. J. E 2005, 16, 253.

(25) Petukhov, A. V.; van der Beek, D.; Dullens, R. P. A.; Dolbnya, I. P.; Vroege, G. J.; Lekkerkerker, H. N. W. Phys. Rev. Lett. 2005, 95, 077801.
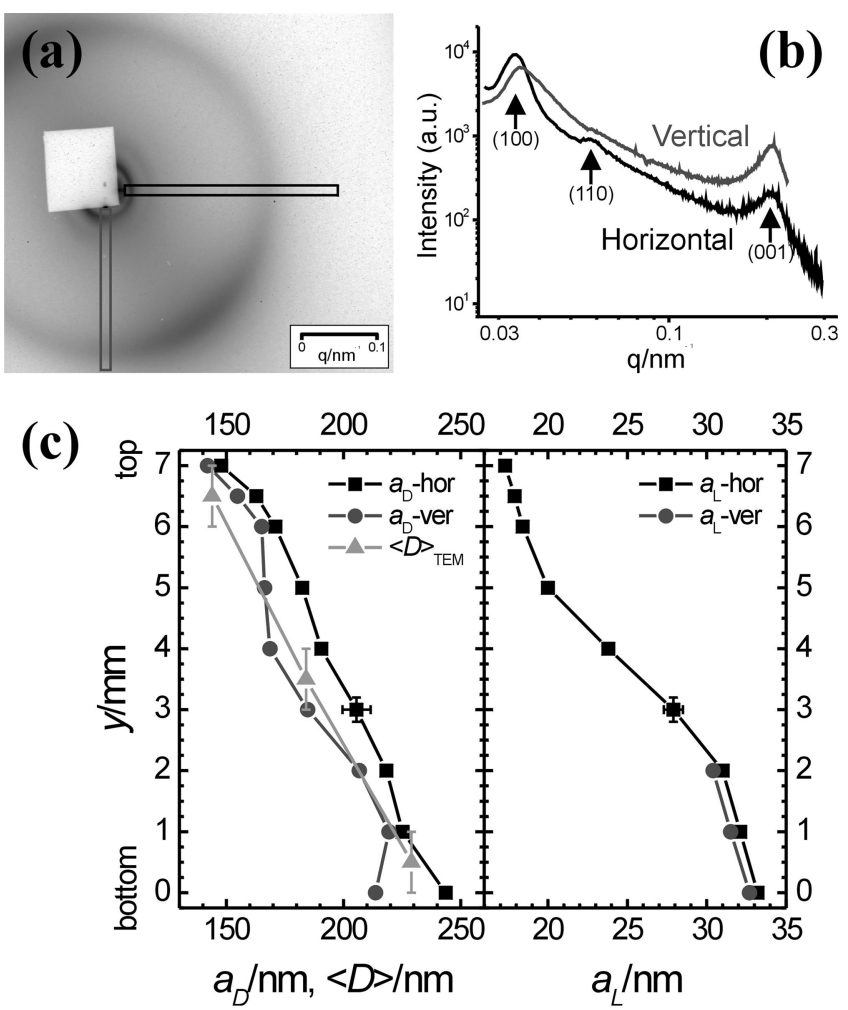

Figure 2. Small-angle $X$-ray scattering analysis of the layers in the columnar phase grown at $900 \mathrm{~g}$. We have measured the periodicity of the columnar crystal as a function of height in the sediment from scattering patterns such as the one shown in part a, which was obtained at a height of $y=2 \mathrm{~mm}$. (b) Averaged radial intensity (for the rectangular slices depicted in part a) as a function of scattering vector $q$ in both the horizontal and vertical directions. The full dependency of lattice spacing vs height is depicted in part $\mathrm{c}$ and $\mathrm{d}$, where we show $a_{D}$ (from small $q$ ) and $a_{L}$ (from large $q$ ), respectively, for the horizontal $(\boldsymbol{\square})$ and vertical $(\boldsymbol{O})$ directions as well as the average particle diameter $\langle D\rangle(\mathbf{\Delta})$ obtained from transmission electron microscopy, as further clarified in Figure 3. $\left(a_{D}, a_{L}\right.$, and $\langle D\rangle$ are defined in the text.)

diameter (thickness). One of the paradigms in colloid science is the notion that the inherent polydispersity of colloids suppresses the formation of ordered phases. For spheres, the polydispersity was demonstrated to slow down the crystallization rate significantly, ${ }^{26}$ and for a polydispersity exceeding the so-called terminal polydispersity of $5-10 \%$, spherical colloids refuse to crystallize. ${ }^{27}$ Similarly, colloidal rods no longer fit into the layers of smectic liquid crystals at more than $18 \%$ length polydispersity. ${ }^{28}$ The recent observation of Vroege and co-workers ${ }^{17}$ of smectic liquidcrystalline order in suspensions of highly polydisperse goethite nanorods shows that the polydispersity issue is rather subtle and can include effects of particle fractionation, sedimentation kinetics, and the coexistence of different ordered phases. In the case of gibbsite platelets, the solution of the polydispersity problem might be related to the formation of an intermediate phase with hexatic-like ordering that has a finite positional correlation length. ${ }^{25}$ This phase may be an intermediate state that significantly reduces the crystallization barrier, or it may even be stabilized by particle polydispersity. In this work, we show that polydisperse gibbsite platelets not only form hexagonal

(26) Pusey, P. In Liquids, Freezing, and the Glass Transition; Hansen, J. P., Levesque, D., Zinn-Justin, J., Eds.; North-Holland: Amsterdam, 1991; Vol. 2, pp 763-931.

(27) Bolhuis, P. G.; Kofke, D. A. Phys. Rev. E 1996, 54, 634. Bartlett, P.; Warren, P. B. Phys. Rev. Lett. 1999, 82, 1979.

(28) Bates, M. A.; Frenkel, D. J. Chem. Phys. 1998, 109, 6193. 

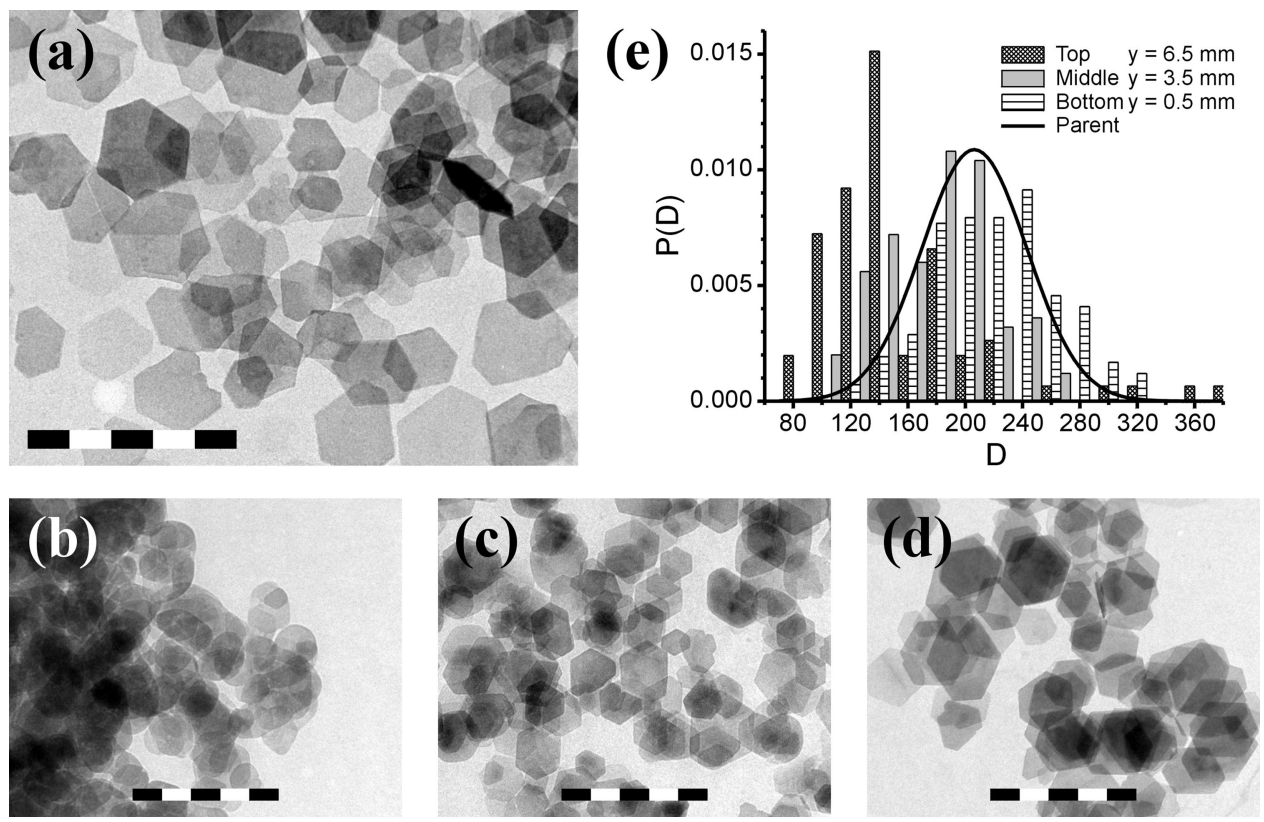

Figure 3. Transmission electron microscopy analysis of three layers of the sediment. The parent suspension is shown in part a, whereas parts $\mathrm{b}-\mathrm{d}$ depict the top $(y=6.5 \mathrm{~mm})$, middle $(y=3.5 \mathrm{~mm})$, and bottom $(y=0.5 \mathrm{~mm})$ layers, respectively. In each graph, the scale bar denotes $500 \mathrm{~nm}$. The micrographs already hint at a pronounced fractionation of the particles, as confirmed by measurement of the average particle diameter in each layer and shown in part e.

columnar phases under slow sedimentation but also form this iridescent phase in less than 1 day under a centrifugal force of $900 g$.

Following a procedure developed earlier in our laboratory, a suspension of hexagonal gibbsite platelets was synthesized. ${ }^{29,23}$ A cylindrical glass tube was filled with the suspension and left undisturbed under normal gravity. The suspension, which has a milky appearance due to light scattering of the freely rotating platelets, develops a sediment in years. It shows strong iridescent colors (Figure 1a,b) as a result of Bragg reflections by the columnar liquid-crystalline order. ${ }^{23}$ Close inspection of the sediment reveals two different layers, sketched in Figure 1c. The first (lower) one was formed relatively quickly (in a few months) and has a turbid appearance, although a few iridescent spots can be observed. In contrast, the top layer takes much longer to form, shows large, millimeter-sized iridescent domains, and appears more translucent. The growth rate of this second layer is a very low (average) $1 \AA / \mathrm{s}$, as measured by the height increase in time. Currently, the sedimentation process is not finished, and the iridescent layer continues to grow. The slow growth rate of our sample as well as its striking iridescence reminds one of the formation of opal. ${ }^{11}$ From a practical point of view, it would be worthwhile to test whether it is possible to speed up this "synthetic columnar opal production". Interestingly, this appears to be the case if the concentration is increased rapidly by centrifugation.

Before applying centrifugation, we have enhanced the particles' colloidal stability by treatment with $\mathrm{Al}_{13}$ Keggin ions. From previous studies, it appears that the presence of these $\mathrm{Al}_{13}$ ions has a stabilizing effect on colloidal boehmite $(\mathrm{AlO}(\mathrm{OH}))$ and gibbsite particles, ${ }^{21,30,31}$ although it limits our study in that a direct comparison between the suspensions is not possible. The centrifuged sediment displays bright violet and green iridescent

(29) Wierenga, A. M.; Lenstra, T. A. J.; Philipse, A. P. Colloids Surf., A 1998 134,359 .

(30) van Bruggen, M. P. B.; Donker, M.; Lekkerkerker, H. N. W.; Hughes, T. L. Colloids Surf., A 1999, 150, 115.

(31) Bugosh, J. J. Phys. Chem. 1961, 65, 1789. Ramsay, J. D. F.; Daish, A. R.; Wright, C. J. J. J. Chem. Soc., Faraday Discuss. 1978, 65, 65. Hernandez, J. Thèse de Doctorat de l'Université Pierre et Marie Curie, Université Pierre et Marie Curie, Paris, France, 1998. colors (Figure 1d) and appears to consist of numerous small crystallites, which we attribute to the fast formation as in the early stage of the first sample (Figure 1a,b).

Under high gravitational forces, one expects a compression of the sediment, resulting in a gradient in the periodicity of the Bragg reflections, with smaller spacings on going down the sediment. In turn, this becomes evident through the colors of the sediment: the smaller the separation between the particles, the smaller the wavelength of the reflected light. This exact phenomenon has been observed in suspensions of the centrifuged Tipula iridescent virus that exhibited Bragg reflections with "a smooth gradation in color ranging from violet at the bottom through green to red at the top". ${ }^{5}$ In the present sample, the order is reversed: violet appears at the top, and green and even some red are found below. To shed light on this issue, we have analyzed the sediment with small-angle X-ray scattering (SAXS) and transmission electron microscopy (TEM).

The SAXS study shows correlation peaks at low and high scattering vector $q$. Figure 2 a shows a typical example of a scattering pattern, and Figure $2 b$ shows radial intensity averages in the vertical and horizontal directions. The columnar identity of the liquid-crystalline sediment is confirmed by the relative peak spacings, indicated by their Miller indices $(h k l) .{ }^{22,24,25}$ From the ringlike diffraction and the fact that both the (100) and (001) peaks are visible, we infer powder diffraction, corroborating our earlier observation of small crystallites. Lattice spacings $a_{D} \equiv$ $4 / 3 \sqrt{3} \sqrt{h^{2}+h k+k^{2}} / q_{(h k 0)}$ and $a_{L} \equiv 2 \pi / q_{(001)}$ are plotted as a function of height in Figure 2c. This variation is in agreement with our observation that the colors are in the order opposite to that expected from gravitational compression.

Our SAXS results are confirmed by an analysis of the particle size distributions at different heights in the sediment using TEM. A concentrated sample was placed in a narrow plastic cell and centrifuged. It was subsequently immersed in liquid nitrogen to freeze it instantaneously. The sediment was cut into 1-mm-thick slices, and care was taken to avoid melting the sample. Three layers, taken at heights $y=0.5,3.5$, and $6.5 \mathrm{~mm}$, were imaged with TEM. From the micrographs (of which typical examples 
are shown in Figure 3), the average particle diameter $\langle D\rangle$ and polydispersity $\sigma_{D}$ were determined for the three layers as well as the parent suspension.

On the TEM graphs, one immediately observes size fractionation in the sediment. The top layer contains the smallest particles, and the middle and lower layers show an increasing average diameter. Thus, the columnar periodicity increases on going down in the sediment, directly causing the iridescent color inversion. The histogram in Figure 3e quantitatively confirms this conclusion, and from Figure $2 c$, it appears that the average diameter perfectly agrees with the (intercolumnar) lattice spacings obtained from SAXS. However, the intracolumnar spacing $a_{L}$ is much larger than the average particle thickness of $13.2 \mathrm{~nm}$. This observation is in agreement with computer simulations ${ }^{32}$ and theoretical calculations, ${ }^{33}$ which yield a nomalized intercolumnar spacing of $a_{D} / D=1.15$, whereas the normalized intracolumnar spacing is $a_{L} / L=1.63$. This may reflect the $1 \mathrm{D}$ liquid character of the columnar phase within the columns.

The question arises as to whether the sedimentation rates of the differently sized particles account for the observed fractionation. Using the effective friction factor of randomly oriented plates, obtained by Brenner ${ }^{34}$ as $f_{\text {eff }}=6 \eta_{0} D$ where $\eta_{0}$ is the viscosity of the solvent, we calculated the sedimentation velocity of the large and small particles (as found in the bottom and top layer of the sediment, respectively) at infinite dilution, and found 5 and $2 \mu \mathrm{m} / \mathrm{s}$, respectively. Taking into account the sedimentation height of $10 \mathrm{~cm}$, this yields an upper boundary for a sedimentation time of $6 \mathrm{~h}$ for large particles and $14 \mathrm{~h}$ for the small particles.

(32) Zhang, S.-D.; Reynolds, P. A.; van Duijneveldt, J. S. J. Chem. Phys. 2002, 117, 9947.

(33) Wensink, H. H. Phys. Rev. Lett. 2004, 93, 157801.

(34) Brenner, H. J. Multiphase Flow 1974, 1, 195.
This will clearly be sufficient to account for the fractionation observed in our samples. A related issue is the nucleation/ crystallization rate of the columnar phase as the particles form a sediment. Although we have no data available to address this issue in a quantitative way, our results suggest that the nucleation/ crystallization rate is faster than or at least on the order of the sedimentation rate because the sediment is crystalline throughout.

We have shown that one can easily prepare columnar opals of colloidal platelets despite their very high polydispersity. Moreover, the process can be accelerated by orders of magnitude using centrifugation at as much as $900 \mathrm{~g}$ without arresting the system in a disordered glassy phase. The ease of crystallization can be partially related to particle fractionation during centrifugation. In particular, the fractionation leads to an unexpected effect of color inversion in the sediment that strongly opposes osmotic compression. Finally, the formation of the hexatic phase $\mathrm{e}^{25}$ with a finite positional correlation length may play a role in the self-organization of the platelets. The fast and easy formation of synthetic columnar opals opens up new possibilities for the industrial-scale production of ordered mesoscopic materials.

Acknowledgment. We thank Patrick Davidson (LPS, Orsay) for helpful discussions, Soumyajit Roy (Van't Hoff Laboratory, Utrecht) for a critical reading of the manuscript, and the personnel of the DUBBLE beamline (ESRF, Grenoble) for support. The Nederlandse Organisatie voor Wetenschappelijk Onderzoek (NWO) is acknowledged for funding and for granting beam time at DUBBLE.

Supporting Information Available: Experimental details of the synthesis, preparation, and characterization of the suspensions. This material is available free of charge via the Internet at http://pubs.acs.org.

LA7012914 